\title{
Transient Paraplegia in a Patient with Bilateral Posterior Frontal Contusions and Traumatic Thoracic Aortic Dissection
}

\author{
Sonia Teufack, MD; Phi Nguyen, BS²; Atul, Rao, MD³; Jay Jenoff, MD; \\ James Harrop, MD ${ }^{1}$ \\ ${ }^{1}$ Neurosurgery Department, Thomas Jefferson University Hospital, Philadelphia, PA \\ 2Jefferson Medical College, Philadelphia, PA \\ ${ }^{3}$ Vascular Surgery Department, Thomas Jefferson University Hospitals, Philadelphia, PA \\ ${ }^{4}$ General Surgery Department, Thomas Jefferson University Hospitals, Philadelphia, PA
}

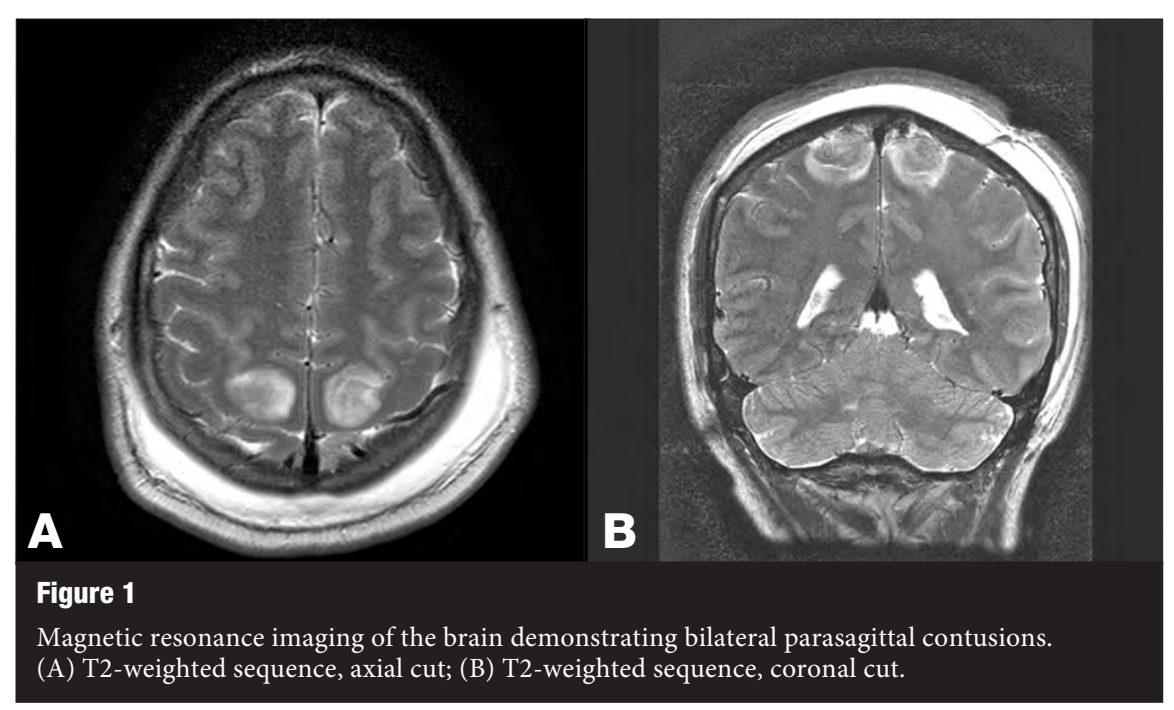

\section{ABSTRACT}

Background: In the setting of multi-system traumas, the etiology and pathophysiology of neurologic injuries can be difficult to identify.

Methods: A unique case of a pedestrian struck by a motor vehicle that presented with acute paraplegia after an endovascular stent placement for a traumatic thoracic aorta dissection. The patient had no significant motor function in the lower extremities, but full preservation of all sensory modalities. Initial admission computed tomography (CT) imaging was negative for intracranial trauma, but noted an acute cranial fracture; no spinal trauma was identified.

Results: The patient had a lumbar drain placed to maximize spinal perfusion pressures and was immediately evaluated with magnetic resonance imaging (MRI) of the neural axis. Acute bilateral posterior frontal contusions were identified on brain imaging, which were not present on preprocedural CT head. No spinal cord injury or ischemia was seen on spinal imaging. The patient recovered and regained use of his lower extremities following a short rehab stay.

Conclusion: In the setting of multi-system trauma, a high level of suspicion should exist for alternative etiologies of neurologic injuries. Thorough neurologic examinations and imaging assessments of the nervous system should be conducted to avoid misdiagnosis and improper management of occult injuries. This is the first reported case of acute paraplegia due to vertex trauma which may be a rare mechanism of injury and/or under-recognized.

\section{INTRODUCTION}

Trauma is the leading cause of mortality for patients less than forty years of agecheck. Further multi-system trauma has been associated with increased mortality, especially when it involves vascular injuries. ${ }^{4}$ Therefore, prompt diagnosis and management is crucial and increases the probability of survival and a favorable outcome.

Acute paraplegia is an associated complication of traumatic thoracic spinal cord and aortic injuries. Further, it can also result from open and endovascular repair of this injury due to decreased perfusion to the cord and ensuing ischemia and infarction. ${ }^{4,5,14}$ Few cases of acute lower extremity monoparesis have been reported from traumatic injury to the frontal lobe. ${ }^{2,10} \mathrm{We}$ present the first case of acute paraplegia resulting from bilateral para-sagittal frontal contusions in a patient with concomitant thoracic aorta injury.

\section{CASE REPORT}

A 31 year-old male pedestrian was struck by a high velocity vehicle and thrown approximately seventy feet from the initial site of impact. The patient was intubated at the scene and transported to an outside hospital. At the time he was following commands and moving all extremities. CT of the brain revealed a bicoronal scalp laceration and underlying skull fracture with no intracranial hemorrhage or contusion; CT of the chest, abdomen and pelvis revealed an aortic isthmus tear, bilateral pneumo-hemothoraxes and pulmonary contusions, multiple ribs and right clavicle fractures, liver laceration with intraperitoneal hemorrhage.

Upon transfer to our facility, the patient remained stable. He would open his eyes to voice, pupils were equal round and reactive, he would follow simple commands in all four extremities with some efforts against gravity. The patient was urgently taken to the operating room for endoscopic repair of the thoracic aortic tear. The procedure was completed without complication, with placement of a Gore TAG $26 \mathrm{~mm} \times 10 \mathrm{~cm}$ endoprosthesis. The patient remained intubated and neurologic 

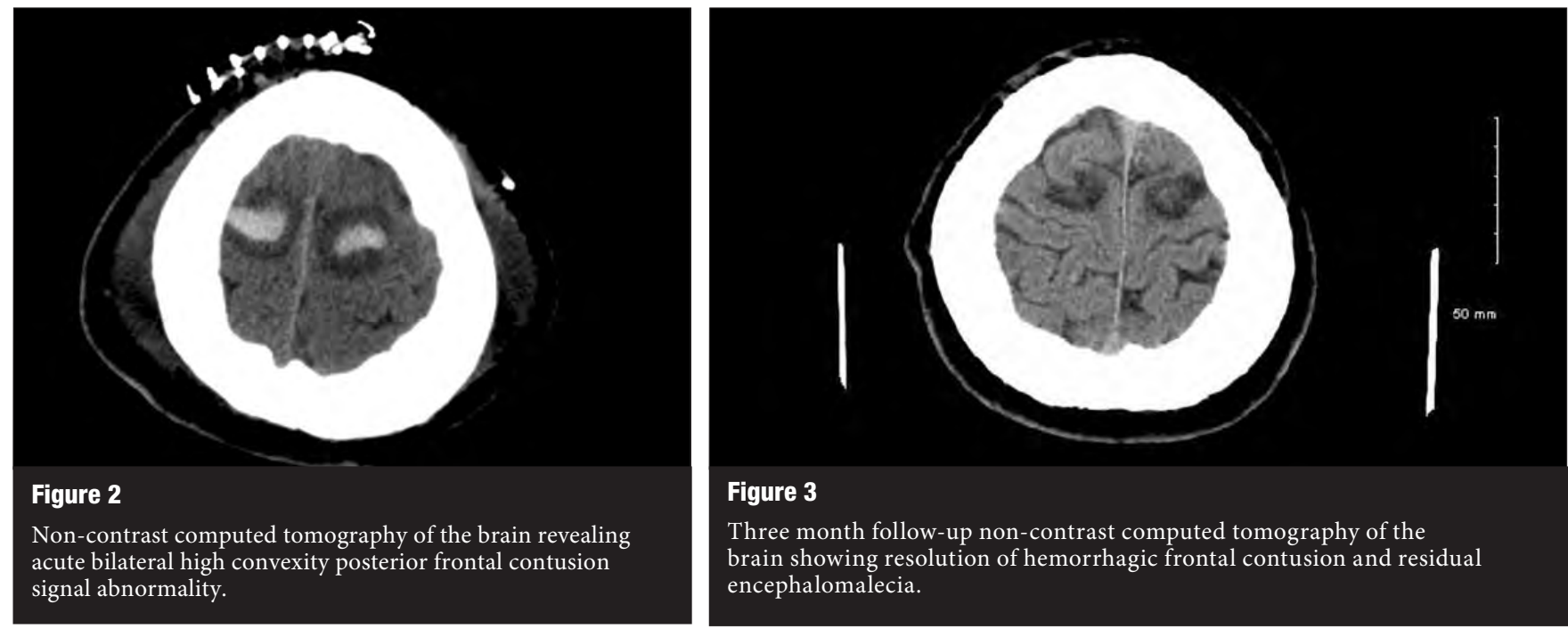

Figure 3

Three month follow-up non-contrast computed tomography of the

brain showing resolution of hemorrhagic frontal contusion and residual encephalomalecia.

examination revealed his upper extremities were $5 / 5$ strength in all tested muscle groups. In the lower extremities he had a questionable right ilio-psoas voluntary muscle twitch; he was otherwise 0/5 in all other muscle groups. Sensory exam revealed normal pinprick, light touch and propioception. The patient had a good rectal tone but no volition.

Due to a concern for post-endovascular repair cord ischemia and infarction, a lumbar drain was inserted to maintain low CSF pressure and maximize spinal perfusion pressures. The patient was evaluated with an MRI of the brain and entire spine. It revealed bilateral high convexity posterior frontal parasagittal contusions underlying the area of scalp laceration (Figures 1 and 2), and a normal cervical, thoracic and lumbar spinal cord and column.

The patient had no further surgical intervention and was discharged to an acute rehab after a couple of weeks of recovery. At his 3 months follow-up, the patient had an almost complete recovery of lower extremity motor strength and was ambulating independently. Follow-up CT head revealed evolution of the contusion with complete resorption of blood products and persistent of edema and encephalomalecia (Figure 3).

\section{DISCUSSION}

Paraplegia can result from a disruption at any level of the neural motor pathway starting from the frontal parasagittal cortical neurons to the thoraco-lumbar spinal cord upper motor neurons, the peripheral lumbar spinal cord roots and peripheral nerves. Post-traumatic acute paraplegia most commonly results from a thoracic spinal cord column fracture and spinal cord injury or vascular injury to the thoracic aorta. Aortic trauma accounts for less than $0.5 \%$ of all trauma admissions, but it is the second leading cause of trauma-related death, second to head trauma. ${ }^{4} \mathrm{Up}$ to $90 \%$ of patients suffering blunt aortic injury (BAI) expire before reaching a hospital; and of those that do, another half dies within 24 hours. ${ }^{13,8,11,9}$ BAI also carries a significant morbidity, including spinal cord ischemia and stroke reported as high as $20 \%$ and $8 \%$ respectively. ${ }^{5}$ Spinal cord ischemia can be the direct result of aortic injury or can be the complication of open and endovascular repairs of the aortic injury. This is thought to be the result of decreased or insufficient blood flow to a susceptible area of the thoracic spinal cord. Endovascular repairs have been shown to have a significantly lower incidence of paraplegia, by avoiding aortic cross-clamping (results in increased CSF pressure and decreased distal aortic perfusion). ${ }^{14}$

This patient presented with an isthmic aortic tear and underwent an emergent endovascular repair. Post-operatively, his paraplegia was initially postulated to result from a spinal cord ischemia due to lose of spinal cord perfusion. A lumbar drain was placed to maintain a low intra-spinal CSF pressure and improve cord perfusion. The patient did not have any improvement from the lumbar drainage. Further assessment with an MRI failed to show any evidence of spinal cord ischemia or stroke, but did note the bilateral cerebral contusions in the primary motor cortex (Figures 1 and 2). It is postulated that the direct trauma to the patient's skull and brain parenchyma manifested as a skull fracture was the etiology of the neurologic deterioration. As part of the endovascular procedure, the patient was anticoagulated with heparin. Therefore we suspect a hemorrhagic conversion or evolution of previously small cerebral contusions resulted in his lower extremity weakness. ${ }^{1,7}$ Venous infarction from superior sagittal sinus thrombosis was excluded by a negative brain MRI.

Paraplegia from cerebral contusion is extremely rare, and to the best of our knowledge has not been previously reported. This rarity of literature might reflects the fact that only $\sim 4 \%$ of all cortical contusions occur in the superior fronto-parietal cortex ${ }^{3,6}$; accordingly fewer would happen in the specific para-sagittal cortex responsible for lower extremity movement. Atac et al. ${ }^{2}$ reported a case of left foot drop secondary to a cerebral contusion from a gunshot wound. Lega et al. ${ }^{10}$ also reported two cases of lower extremity monoparesis due to traumatic intraparenchymal contusions. They made the point that conventional axial CT imaging does not always provide adequate imaging of the vertex, primarily because of volume averaging. In this case one may speculate that our patient's initial CT failed to detect small vertex contusions. Our recommendation is for a high level of suspicion in patients with external signs of cranial traumas and improved screening with high resolution axial CT and coronal reconstruction.

\section{CONCLUSION}

In the setting of multi-system trauma, a high level of suspicion should exist for multivariable injuries. Acute paraplegia due to vertex trauma is a very rare injury but should be included in 
the differential for a patient with bilateral lower extremity weakness and negative MRI of the spinal axis.

\section{REFERENCES}

1. Amabile P, Collart F, Gariboldi V, Rollet G, Bartoli JM, Piquet P: Surgical versus endovascular treatment of traumatic thoracic aortic rupture. J Vasc Surg 40:873-879, 2004

2. Atac K, Ulas UH, Erdogant E, Gokcil Z: Foot drop due to cranial gunshot wound. Mil Med 169:568-569, 2004

3. Besenski N: Traumatic injuries: Imaging of head injuries. Eur Radiol 12:1237-1252, 2002

4. Clancy TV, Gary Maxwell J, Covington DL, Brinker CC, Blackman D: A statewide analysis of level I and II trauma centers for patients with major injuries. J Trauma 51:346351,2001
5. Clouse WD: Endovascular repair of thoracic aortic injury: Current thoughts and technical considerations. Semin Intervent Radiol 27:55-67, 2010

6. Gentry LR, Godersky JC, Thompson B: MR imaging of head trauma: Review of the distribution and radiopathologic features of traumatic lesions. AJR Am J Roentgenol 150:663672,1988

7. Jahromi AS, Kazemi K, Safar HA, Doobay B, Cina CS: Traumatic rupture of the thoracic aorta: Cohort study and systematic review. J Vasc Surg 34:1029-1034, 2001

8. Jamieson WR, Janusz MT, Gudas VM, Burr LH, Fradet GJ, Henderson C: Traumatic rupture of the thoracic aorta: Third decade of experience. Am J Surg 183:571-575, 2002

9. Kwon CC, Gill IS, Fallon WF, Yowler C, Akhrass R, Temes $\mathrm{RT}$, et al: Delayed operative intervention in the management of traumatic descending thoracic aortic rupture. Ann Thorac Surg 74:S1888-91; discussion S1892-8, 2002
10. Lega BC, Bailey RL, Kramer DR, Heuer GG, Chen JY, Schuster JM: Isolated lower extremity monoplegia due to a traumatic intraparenchymal contusion: Report of two cases. Brain Inj 24:1231-1235, 2010

11. Lin PH, Bush RL, Zhou W, Peden EK, Lumsden AB: Endovascular treatment of traumatic thoracic aortic injury-should this be the new standard of treatment? J Vasc Surg 43 Suppl A:22A-29A, 2006

12. MacKenzie EJ FC: Epidemiology, in Moore EE, Feliciano DV, Mattox KL: Trauma, New York, NY: McGraw-Hill, 2004, pp 28-37

13. Plummer D, Petro K, Akbari C, O'Donnell S: Endovascular repair of traumatic thoracic aortic disruption. Perspect Vas Surg Endovasc Ther 18:132-139, 2006

14. Schumacher H, Bockler D, von Tengg-Kobligk H, Allenberg JR: Acute traumatic aortic tear: Open versus stent-graft repair. Semin Vasc Surg 19:48-59, 2006

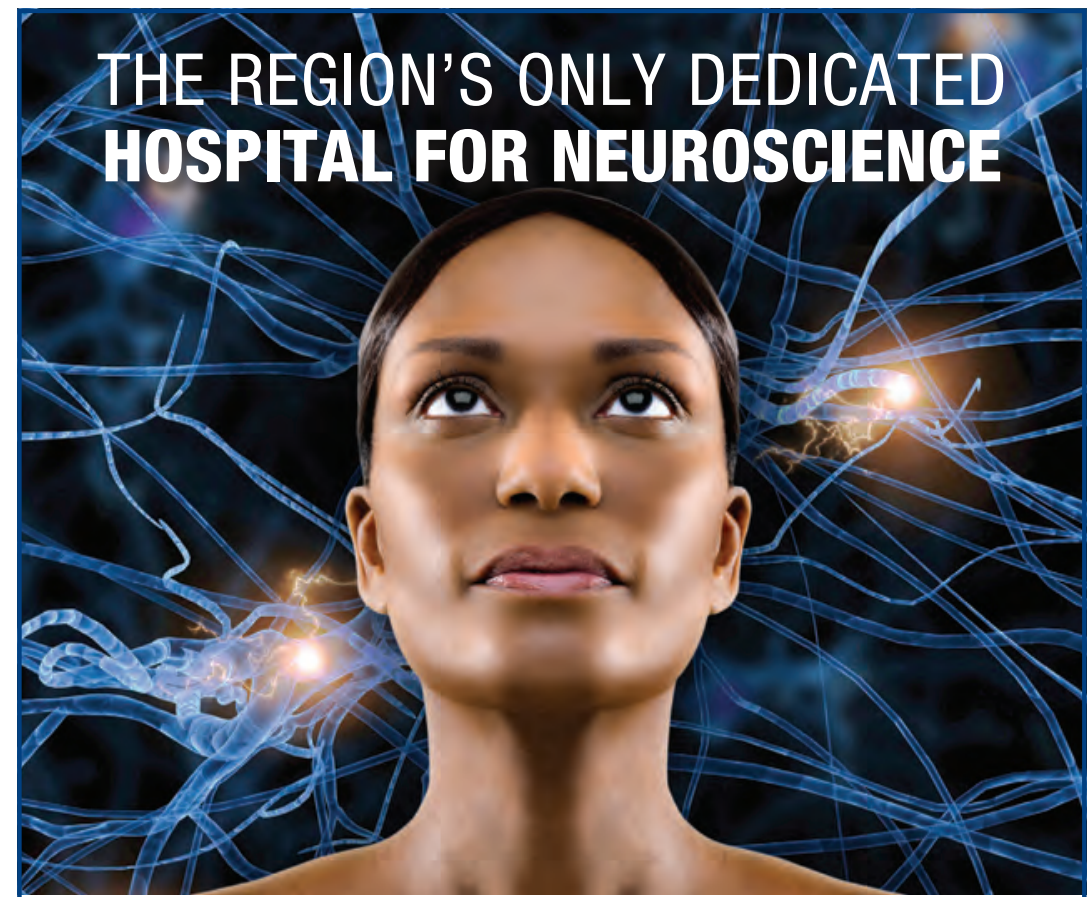

Jefferson Hospital for Neuroscience is the region's only hospital dedicated to neurosurgery and neurology, with specialized critical care units and advanced technology. The experience of our physicians - including six board-certified neurointensivists - and nurses is nearly impossible to match, especially when it comes to treating brain aneurysms, AVMs, brain tumors and stroke with minimally invasive procedures they helped to develop. For neuroscience, experience the advantages that choosing our team makes. Choose Jefferson.

\section{Sefferson.}

Hospital for Neuroscience
- Thomas Jefferson University Hospital - Jefferson Hospital for Neuroscience - Methodist Hospital

1-800-JEFF-NOW 1-800-533-3669 